\title{
Resektables Pankreaskarzinom \\ Welchen Einfluss hat der Resektionsstatus auf die Prognose?
}

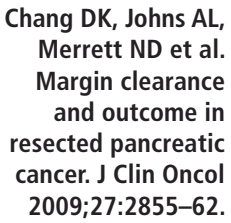

\section{Fragestellung und Hintergrund}

Auch in Deutschland gehört das Pankreaskarzinom mit dem duktalen Adenokarzinom mittlerweile zu den weitaus häufigsten Malignomen mit etwa 13000 Neuerkrankungen pro Jahr. Allerdings haben nur etwa $20 \%$ der betroffenen Patienten ein resektables Karzinom, und selbst nach einer Tumorresektion „,im Gesunden“ (R0) betragen das mediane Überleben nur 12-22 Monate und die 5-Jahres-Überlebensrate allenfalls $20 \%$. Die Autoren gingen deshalb dem Zusammenhang zwischen Tumornähe zum Resektionsrand und der Prognose nach.

\section{Patienten und Methodik}

Acht Kliniken analysierten über 17 Jahre hinweg überwiegend retrospektiv die von ihnen operierten duktalen Adenokarzinome. In dieser Zeit wurden 295 partielle Duodenopankreatektomien (Rechtsresektionen) und 70 Linksresektionen durchgeführt. Dabei wurde offenbar systematisch auch der zirkumferenzielle Resektionsrand untersucht.

\section{Ergebnisse}

Nach einem medianen Nachbeobachtungszeitraum von 15,6 Monaten lebten noch $14,8 \%$ der operierten Patienten. Die 3-Jahres-Gesamtüberlebensrate betrug $23,8 \%$, die 5-Jahres-Gesamtüberlebensrate $11,4 \%$. Eine R0-Resektion war nur in $63,8 \%$ erreicht worden. Die R1-Resektion war ein unabhängiger, prognostisch negativer Faktor. Bei näherer Analyse des tumorfreien zirkumferenziellen Sicherheitsabstands war die Prognose zusätzlich signifikant besser, wenn dieser mindestens $2 \mathrm{~mm}$ betrug.

\section{Schlussfolgerung}

Beim lokal begrenzten Pankreaskarzinom ist das Erreichen einer R0-Resektion ein wichtiger Prognosefaktor. Bei knappen Sicherheitsabständen muss postoperativ bestrahlt werden.

Schlüsselwörter: Pankreaskarzinom - Resektionsstatus - Prognose

\section{Eine neoadjuvante Radiochemotherapie ist meist sinnvoll Kommentar von Werner Hohenberger, Erlangen}

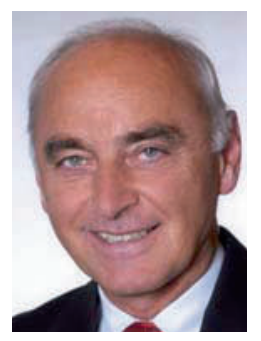

Prof. Dr. med.

Werner

Hohenberger Direktor der

Chirurgischen Klinik des Universitätsklini-

kums Erlangen.

E-Mail: werner.

hohenberger@chir. imed.uni-erlangen.de
D ie Schlussfolgerung der Autoren, dass die Patienten bei einem Sicherheitsabstand $<1,5 \mathrm{~mm}$ postoperativ eine Strahlentherapie erhalten sollten, ist zunächst konsequent. Anhand der mittlerweile vorliegenden Ergebnisse beim Rektumkarzinom und auch im Hinblick auf frühere Publikationen zur adjuvanten Radiotherapie muss man jedoch diese Forderung schon allein deshalb infrage stellen, weil man beim primär resektablen Pankreaskarzinom in der Regel keine wirkliche R0-Resektion erreicht, obwohl der histopathologische Befund dies nahelegt. Vielmehr lässt sich feststellen, dass man anhand einer exakten bildgebenden Diagnostik bereits präoperativ den sich im Fall einer Resektion ergebenden zirkumferenziellen Sicherheitsabstand abschätzen sollte. Ist absehbar, dass dieser Abstand um $2 \mathrm{~mm}$ unterschritten wird, sollte der Operation eine neoadjuvante Radiochemotherapie vorgeschaltet werden. Aufgrund der engen Beziehungen des Pankreas zu den umgebenden Gefäßen trifft dies auf die überwiegende Mehrzahl der duktalen Adenokarzinome des Pankreas zu [1]. Der von der Heidelberger Gruppe zum Rektumkarzinom publizierte Beitrag sollte analog ganz klar als Konsequenz nach sich ziehen, dass nahezu jedes duktale Pankreaskarzinom neoadjuvant durch Radiochemotherapie behandelt werden muss.

Entsprechend verfahren wir bereits seit fast 15 Jahren in der eigenen Arbeitsgruppe. Um dies auch wissenschaftlich zu belegen, vergleichen wir in einer seit einigen Jahren laufenden randomisierten Studie die primäre Operation mit der Resektion nach neoadjuvanter Radiochemotherapie. Diese Studie werden wir Ende des Jahres schließen und dann über die Ergebnisse berichten.

\section{Literatur \\ 1. Esposito I et al. Ann Surg Oncol 2008;15:1651-60.}

[Erstmals publiziert in: Strahlenther Onkol 2009;10:704.] 